\title{
STRUCTURAL MRI MEASURES IN ALZHEIMER'S DISEASE AND EFFECTS OF ETHNICITY
}

Linda Zhang ${ }^{1}$, Raymond Tak-Fai Cheung ${ }^{1}$, Henry Ka Fung Mak ${ }^{1}$, Leung-wing Chu ${ }^{2}$

${ }^{1}$ University of Hong Kong, Hong Kong, Hong Kong;

${ }^{2}$ The University of Hong Kong, Hong Kong, Hong Kong.

BACKGROUND: Alzheimer's disease (AD) affects populations worldwide, and there are increasing numbers of $\mathrm{AD}$-focused international neuroimaging studies. For these global cohorts, ethnicity may be a confounding factor. Morphometric differences have been found between European and Japanese brains, though the implications for common AD neuroimaging biomarkers, such as hippocampal volume and cortical thickness, are unknown and should be investigated. METHODS: 65 local Chinese participants (33 AD, 32 elderly controls) were recruited, and a matching Caucasian cohort was downloaded from ADNI. All participants underwent T1-weighted MPRAGE scanning with ADNI's protocol in 3T Philips Achieva scanners, and the raw images were processed using FreeSurfer v5.1. Cortical thickness was compared between groups with FreeSurfer's GLM analysis tools. Hippocampal volumes were corrected for intracranial volume and analysed using multivariate GLM, with age, gender and years of education as covariates. Results: There were no significant differences between the two ethnicities for age, gender, MMSE and ADAS-cog scores, but local Chinese had significantly fewer years of education than Caucasians (4.863.8 vs. 15.863.3). Several clusters in the bilateral cingulate and precuneus regions showed cortical thinning in Chinese patients whereas Caucasian patients only had thinning in the inferior temporal regions compared to their respective controls. Between ethnicities, clusters in the left parietal area were thicker in Caucasian patients, whereas Chinese controls had thicker paracentral cortices than Caucasian controls. When combined, patients had cortical thinning in the bilateral temporal regions (all results significant at $\mathrm{p}<0.01$ ). Controls had larger relative hippocampal volumes than patients $(0.2760 .05$ vs. 0.1960 .04$)$, and Chinese subjects had larger relative hippocampal volumes than Caucasian subjects, regardless of diagnosis ( 0.2860 .05 vs. 0.2360 .03 for controls, 0.2060 .04 vs. 0.1760 .02 for patients).

CONCLUSIONS: Chinese subjects have larger relative hippocampal volumes than Caucasian subjects, but Chinese $\mathrm{AD}$ patients also display a greater degree of hippocampal atrophy and cortical thinning. As none of these trends are reflected in the cognitive test scores, this may be indicative of a protective effect from lifestyle, despite fewer years of education. Future analyses could include a cohort of North American Chinese subjects. If the effect is persistent, ethnicity should be taken into account in global population studies. 